\title{
Distinctive shapes benefit short-term memory for color associations, but not for color
}

\author{
Jean-François Delvenne \\ University of Leeds, Leeds, England \\ AND \\ KEVIN DENT \\ University of Birmingham, Birmingham, England
}

\begin{abstract}
In four experiments, we examined the effect of pairing colors with either homogeneous or heterogeneous shapes on a short-term memory task. In Experiment 1, we found no differences in color memory for displays in which colors were each associated with different shapes, paired with individual homogeneous shapes, or paired with heterogeneous shapes. In contrast, in Experiment 2, we found that when participants were asked to remember the specific pairings of colors, memory was improved for heterogeneous-shape displays. The benefit for heterogeneous shapes appears to be memorial, rather than one that occurs at the time of encoding (Experiment 3) or retrieval (Experiment 4). The present study suggests that distinctive shapes can be used to help bind color associations in visual short-term memory.
\end{abstract}

Visual short-term memory (VSTM) is the system responsible for the temporary maintenance of visual information. It is widely accepted that VSTM is extremely limited in capacity, perhaps holding information about only four items at any one time (Irwin, 1992; Luck \& Vogel, 1997; Pashler, 1988; Sperling, 1960). What remains to be determined, however, is the detailed nature of the visual representations employed by VSTM. Several lines of evidence point to the idea that information might be stored in VSTM in the form of integrated object representations. For example, observers are able to store both the colors and orientations of several objects almost as accurately as the colors or the orientations alone, indicating that the number of objects - rather than individual features - limits VSTM capacity (Lee \& Chun, 2001; Luck \& Vogel, 1997; Vogel, Woodman, \& Luck, 2001). Features of objects also tend to be retained or lost from memory together as a unit, rather than independently (Irwin, 1992; Irwin \& Andrews, 1996). Representations in VSTM also code how features were associated together-for example, what went together (Delvenne \& Bruyer, 2004; Wheeler \& Treisman, 2002).

Most studies of VSTM have been concerned with objects composed of a single part. However, in some studies, researchers have begun to consider how more complex multipart objects may be represented in memory. Xu (2002a, 2002b) demonstrated an "object-based encoding benefit" for multipart objects. Memory for a set of part elements was improved considerably when those parts were conjoined together to form single objects. However, $\mathrm{Xu}(2002 \mathrm{~b})$ also demonstrated important constraints on this object-based benefit; namely, it was absent when the parts consisted of features from the same dimension. In the experiments of most relevance to the present study, $\mathrm{Xu}(2002 \mathrm{~b})$ asked people to remember displays composed of caps and stems. When these parts carried different features (color and orientation), there was a benefit from conjoining the elements to form mushroom-like objects. However, when both of the parts carried color features or orientation features, the benefit was absent. One plausible interpretation of these findings is that object representations in memory may store no more than one value for each feature dimension. However, such a conclusion would be premature without further detailed exploration of memory for two colored objects; are there circumstances under which colors can benefit from being conjoined into a single object?

In the present article, we explore memory for objects with different colored parts. In particular, we were interested in how the association of multiple colors is represented in short-term memory. In models of object recognition, it is routinely proposed that when we recognize objects, we make use of hierarchically organized structural descriptions (see, e.g., Marr \& Nishihara, 1978). At the top level of the hierarchy, global shape may be represented, with more detailed information about parts becoming available as the hierarchy is descended. It is certainly the case that shape is the primary means by which different objects are differentiated from one another in object recognition, with color and surface detail playing an important but secondary role (see Biederman \& Ju, 1988; Price \& Humphreys,

J.-F. Delvenne, j.f.delvenne@leeds.ac.uk 
1989 , for a discussion of these issues). Shape provides a powerful means by which long-term visual memory is indexed and organized, and, of course, this makes good sense because shape is much more diagnostic of identity than is color (see Schyns, 1998). There is also good neuropsychological (Humphreys, 2001; Humphreys, Cinel, Wolfe, Olson, \& Klempen, 2000) and psychophysical (Clifford, Holcombe, \& Pearson, 2004) evidence for shape primacy in earlier stages of perceptual analysis; binding of local shape elements into global shapes appears to precede the binding of shape and surface detail. It may well be the case that shape is also primary in VSTM and that global shape provides a powerful cue for the organization of representations in this store.

The present experiments investigated the role of global shape as a potential organizing principle for color VSTM. If objects are defined and differentiated from one another primarily on the basis of shape, then color VSTM capacity could be improved by global shape differences. Previous studies of object-based coding benefits for color $(\mathrm{Xu}$, 2002a, 2002b) have only examined color-color objects that all have the same shape in the display. If object representations in VSTM are organized hierarchically and differentiated primarily by global shape, then it is no surprise that there is no object-based benefit under these conditions. If the color-color objects can be differentiated in terms of shape, then perhaps more colors could be stored, and object-based benefits could be found.

Previous research (e.g., Kanwisher, 1987) has also demonstrated that people often struggle to effectively deal with stimulus repetition. In rapidly presented streams of stimuli, participants often fail to report the second appearance of a target occurring in close temporal succession to the first-a phenomenon known as repetition blindness. Kanwisher proposed that repetition blindness occurs because individuating multiple tokens of the same representational type is a tight, capacity-limited process. Displays of multiple objects with identical shape might similarly tax mechanisms of "tokenization." Adding shape differences might ease the process of creating multiple distinct individual representations, reduce confusions between features, and improve memory performance.

Wheeler and Treisman (2002) pointed out that when considering memory for multifeature objects, it is important for one to distinguish between memory for the features themselves - in this case, the colors - and memory for how these features were associated - for example, which colors went together. In the present experiments, we examined the influence of distinctive shapes on memory for both the colors and the color-color associations present in a set of two-colored objects. In order to make this assessment, we used stimuli composed of colored elements that could be combined into two-part objects that were either identical or different in shape. All experiments examined participants' ability to detect changes to an array of such stimuli made after a delay. Experiment 1 looked at memory for color by asking participants to detect a color change between two arrays. Experiments 2-4 looked at memory for color-color associations by asking participants to detect a change in the association between colors.

\section{EXPERIMENT 1}

In Experiment 1, participants were asked to remember a set of colored equilateral triangles. Participants viewed a set of such triangles, and, a short time later, they decided whether a test display was identical or different in the color of one triangle. The triangles were either spatially separated in the display (separated features display) or combined into pairs of spatially abutting triangles. When paired together, the triangles could form objects with one of three possible shapes. The homogeneously shaped objects displays contained objects that all had the same shape, whereas the heterogeneously shaped objects displays were created using all three possible shapes in a single display. If shape is an important organizing principle for object representations in memory (so that hierarchical object representations are distinguished primarily by global shape), then memory for color should be best when the colors are paired into distinctive shapes.

\section{Method}

Participants. Fourteen participants who were naive to the experimental purpose were tested ( 9 females; mean age $=20.8$ years; range 19-28 years). All had normal (self-reported) or corrected-tonormal visual acuity and normal color vision.

Apparatus. Visual stimuli were displayed on a 17-in. monitor with a refresh rate of $75 \mathrm{~Hz}$. The experiments were implemented using E-Prime (Psychology Software Tools, Inc.; www.pstnet.com/eprime), and responses were collected from the computer keyboard.

Materials. Equilateral colored triangles were used as stimuli. At a viewing distance of $60 \mathrm{~cm}$, the sizes of the triangles were $0.95^{\circ} \times$ $0.95^{\circ}$ of visual angle. Each triangle could be one of eight possible RGB colors (black, blue, green, pink, red, turquoise, white, and yellow) produced by permutation of the presence ( 255 on the $0-255$ scale) or absence ( 0 on the $0-255$ scale) of red, green, and blue phosphors. The stimuli were displayed against a gray background (127 on the $0-255$ scale of red, green, and blue phosphors) in six possible locations on an imaginary circle with a radius of about $3.05^{\circ}$ from the center of the screen $\left(5.8^{\circ} \times 5.8^{\circ}\right.$ of visual angle $)$, with the objects in a given display separated by at least $3.05^{\circ}$ (center to center). The colors used on the same display were not allowed to repeat.

Procedure. The memory display contained four or six colored triangles; half were presented in their canonical upright orientation, and the other half were inverted. There were three types of memory display (see Figure 1). In the separated features displays, the triangles were all spatially separated. There were three possible ways to combine the triangles to form three possible shapes (a diamond and two distinct parallelograms). In the homogeneously shaped objects displays, every upright triangle was attached to an inverted triangle in order to form two or three objects all with the same shape, with the three possible shapes being used equally often over trials. In the heterogeneously shaped objects displays, the triangles were attached so that each of the three possible shapes were present equally often within a single display.

On each trial, the participants were shown a central fixation point (a small white cross subtending $0.28^{\circ} \times 0.28^{\circ}$ at a viewing distance of $60 \mathrm{~cm}$ ) flashing for $500 \mathrm{msec}$, followed by two white digits (approximately $0.5^{\circ}$ each and separated by a $2.38^{\circ}$ visual angle) for $500 \mathrm{msec}$. After a 1,000-msec blank interval, the memory display was presented for $200 \mathrm{msec}$, tracked by a $900-\mathrm{msec}$ blank interval and then by the test display that remained present until a response key was pressed. The test display contained stimuli in the same locations as in the memory display. The participants were instructed to memorize both the two digits and the stimuli presented in the memory display. The primary task was to indicate as accurately as possible whether the memory and test displays were the same or different. When a change occurred, one color was changed to a new color that 
A

\section{Separated Features}

Memory Display

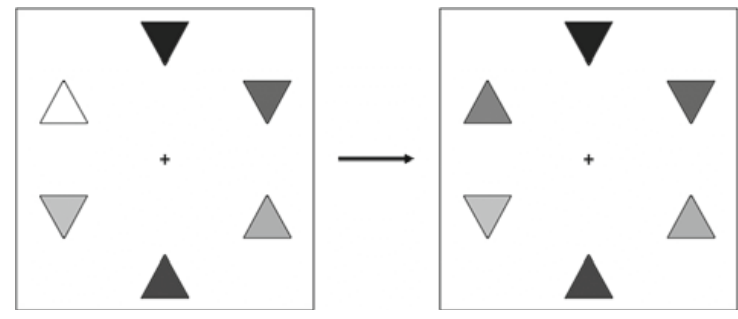

B Homogeneously Shaped Objects

Memory Display

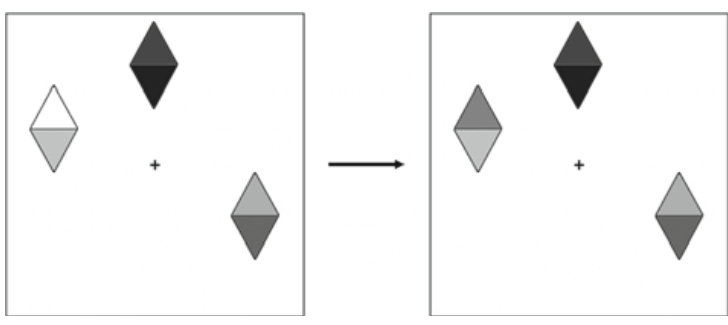

C

Heterogeneously Shaped Objects

Memory Display

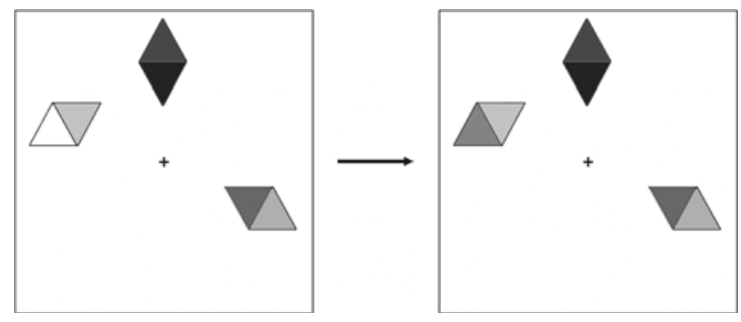

Figure 1. Samples of displays used in Experiment 1. In the separated features display, each colored triangle constituted a spatially separated object. In the homogeneously shaped objects display, the colored triangles were conjoined by twos into similar global shapes; in the heterogeneously shaped objects display, the colored triangles were conjoined by twos into distinct global shapes. The different gray levels represent different colors.

was not present elsewhere in the display. As soon as the participants responded, feedback was given as either a happy schematic face for a correct response or a sad one for an incorrect response. The participants then verbally recalled the two digits displayed at the beginning of the trial and pressed the space bar on the keyboard to generate the next trial starting $1,000 \mathrm{msec}$ later. The verbal memory task was used to prevent the participants from adopting verbal strategies (see Baddeley, 1986). At the end of each block, participants were given their correct response rate and mean response latency, and they were politely warned if their accuracy was lower than $70 \%$. There were breaks between each block during which participants could rest for as long as they wished.

Trials were blocked by display type, for which the order was counterbalanced across participants. For each display type, there were a total of 96 trials, with 48 change trials and 48 no-change trials that were uniformly distributed into three blocks. The participants were tested individually in a quiet room with dim lighting. At the beginning of the experiment, a detailed description of the task was given to the participant. They then completed 12 practice trials to accustom to the stimuli and the task, and 288 experimental trials (i.e., 3 [display types] $\times 2$ [set sizes] $\times 2$ [change vs. no-change trials] $\times 24$ cases).

\section{Results and Discussion}

In the following analysis, we used the nonparametric measure of sensitivity $\left(A^{\prime}\right) .{ }^{1}$ The resulting means of $A^{\prime}$ are plotted in Figure 2. A repeated measures ANOVA tested the effects of display type and set size. The main effect of set size was significant $\left[F(1,13)=206.37, M S_{\mathrm{e}}=0.0001\right.$, $p<.001$, with better performance at set size 4 , but neither the effect of display type $(p>.21)$ nor the interaction between display type and set size $(p>.67)$ were significant. The effects of set size and object type (i.e., diamond, Parallelogram 1, or Parallelogram 2) were also examined in a separate analysis of the homogeneously shaped objects condition only. The repeated measures ANOVA revealed a significant main effect of set size $[F(1,13)=25.525$, $\left.M S_{\mathrm{e}}=0.002, p<.001\right]$, with better performance at set size 4. However, neither the effect of object type $(p>$ $.705)$ nor the interaction between object type and set size $(p>.604)$ were significant.

As in previous studies (e.g., Xu, 2002b), the results failed to show an effect of pairing features from the same dimension (i.e., colors) together on VSTM capacity. Memory performance was indeed the same in the separated features display as it was in the homogeneously shaped and heterogeneously shaped objects displays. The absence of such an object-based encoding benefit in VSTM when the colors were paired into distinctive shapes (i.e., heterogeneously shaped objects displays) is interesting. It would appear that object shape is not a primary feature by which VSTM is organized. Certainly we are able to rule out any model that supposes that capacity in VSTM may be defined in terms of the number of distinctive shapes that may be stored. It is not the case that differences in global shape provide an efficient means for organizing memory that leads to increases in the number of colors that may be stored. Rather, it would seem that color capacity in this context should be defined in terms of the number of colors present.

\section{EXPERIMENT 2}

In the first experiment, we measured the capacity of memory for the individual features. In Experiment 2, we went on to examine participants' knowledge in more detail; in particular, we probed their knowledge of how the colors were associated into objects. Participants were presented with the homogeneously shaped and heterogeneously shaped objects displays from Experiment 1. At test, a single item was presented centrally, and participants decided whether its constituent colors had been paired in the initial display or whether they had originally been in different objects.

We should point out that the extent to which such colorcolor associations can be maintained in memory at all under these brief exposure durations is currently unknown. In 


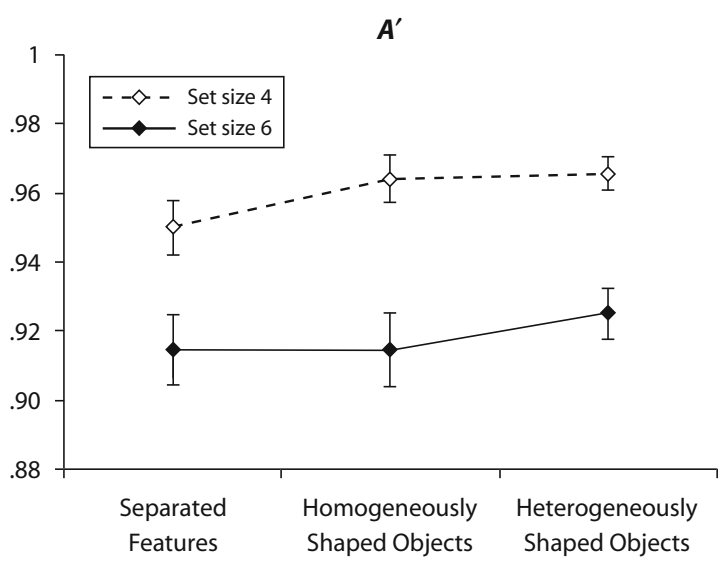

Figure 2. Results of Experiment 1. Error bars represent standard errors of the means.

their initial assessment of conjunction memory, using this conjunction change detection task, Wheeler and Treisman (2002) neglected to consider color-color associations. Assuming that participants can solve this task and maintain color-color associations, it is of interest for one to determine how this is done. It could be that these associations are strong and direct in the sense that colors are associated with each other without reference to other disambiguating information. Alternatively, it might be the case that knowledge of color-color associations makes reference to shape. Associating the component colors of each object with a distinct shape could allow better coregistration and association in memory of the correct pairings. Furthermore, tying the two colors of an object to a distinct shape might provide an additional route for the association of the colors.

If the associations between the colors are strong and direct, then adding shape differences should make no difference. On the contrary, if the color associations are stored with reference to global shape information, then shape differences should matter.

\section{Method}

Participants. Fourteen participants who were naive to the experimental purpose were tested $(11$ females; mean age $=21.1$ years; range 18-30 years). All had normal (self-reported) or corrected-tonormal visual acuity and normal color vision.

Procedure. We used the same stimuli as in the first experiment and two of the three types of memory display: the homogeneously shaped and heterogeneously shaped objects displays. All aspects of the procedure were the same as those in the first experiment, except that only one object was displayed at the center of the screen at test (see Figure 3). The participants were instructed to indicate as accurately as possible whether the test stimulus had been present in the memory display. In all cases, the two colored triangles that formed the test object were present in the memory display; $50 \%$ of the time they had been present in the same combination (present trials), whereas $50 \%$ of the time they were initially present of different objects (absent trials). This procedure forced the participants to memorize not only the colors, but also the associations between them. Doing this allowed us to directly test memory for the associations (Wheeler \& Treisman, 2002). Trials were blocked by display types, for which the order was counterbalanced across participants. After completing 12 practice trials, the participants were given 96

experimental trials (i.e., 2 [display types] $\times 2$ [change vs. no-change trials] $\times 24$ cases $)$

\section{Results}

The results are shown in Figure 4. A paired-samples $t$ test on sensitivity $\left(A^{\prime}\right)$ revealed a significant effect of display type $[t(13)=2.22, p<.05]$, with better performance in the heterogeneously shaped than in the homogeneously shaped objects displays. A separate repeated measures ANOVA also tested the effects of object type (i.e., diamond, Parallelogram 1, and Parallelogram 2) in the homogeneously shaped objects condition. The effect was not significant $\left[F(2,26)=2.559, M S_{\mathrm{e}}=0.017, p>\right.$ .097]. The results showed that participants were able to store in memory which colors went together. Furthermore, memory was improved for heterogeneously shaped objects, indicating that distinctive shapes can be used to help bind color associations in short-term memory.

There are several possible accounts of the shape heterogeneity advantage that was revealed. First, and perhaps most intuitively, shape may provide an additional way to link the colors together, and to prevent erroneous associations between colors from different objects. As such, it may be easier to represent simultaneously several color associations in memory when they are parts of distinct shapes. However, currently, we cannot rule out the possibility that the heterogeneity advantage is perceptual in nature, resulting from greater attentional and perceptual processing difficulty for the homogeneous displays. Several authors (e.g., Duncan \& Humphreys, 1989) have indeed identified display heterogeneity as an important

\section{A Homogeneously Shaped Objects}

Memory Display

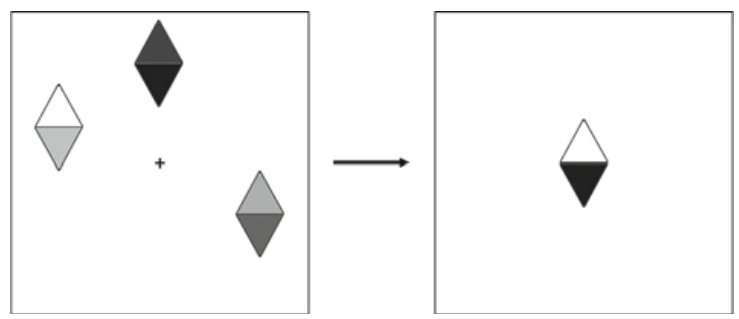

B Heterogeneously Shaped Objects Memory Display

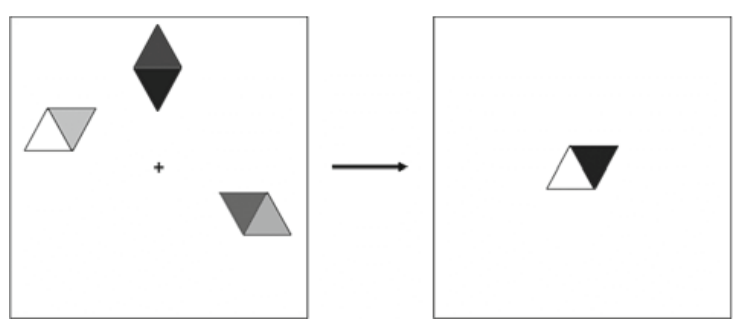

Figure 3. Samples of displays used in Experiment 2. 


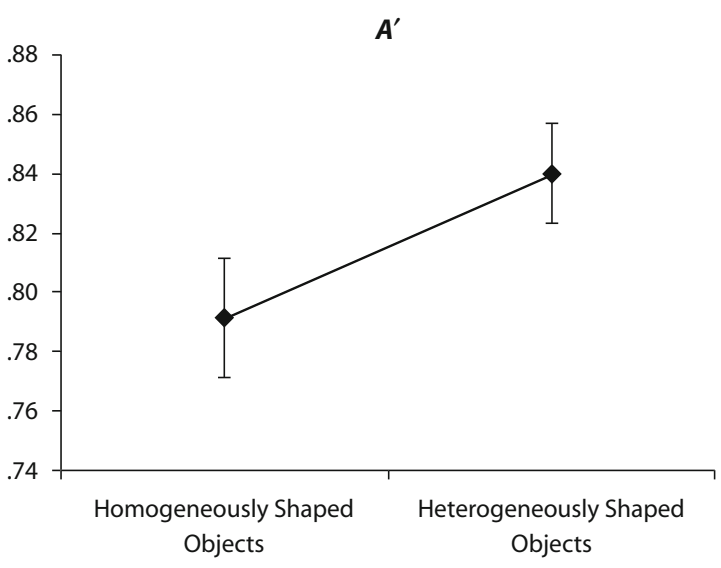

Figure 4. Results of Experiment 2. Error bars represent standard errors of the means.

factor in rapid target selection in visual search. Second, although it seems unlikely — and although none of our participants reported any subjective difficulty in perceiving the displays - we cannot exclude the possibility of more frequent illusory color-color conjunctions (see Treisman $\&$ Schmidt, 1982) in the homogeneous condition. It is also the case that searching through sets of identical objects puts a greater load on spatial memory for where attention has been (Wojciulik, Husain, Clarke, \& Driver, 2001). Alvarez and Cavanagh (2004) demonstrated a relationship between visual search speed and memory capacity, with more complex objects being both more difficult to select as well as more difficult to remember. Although we note that an encoding effect seems unlikely - especially given the null results of Experiment 1-we reasoned that any greater perceptual difficulty in either condition should be reflected in correspondingly greater difficulty in a visual search task, carried out on the same displays.

\section{EXPERIMENT 3}

Experiment 3 employed a visual search task to test for greater interference at the encoding stage for homogeneously shaped as compared with heterogeneously shaped object displays. Importantly, we used exactly the same stimuli as in Experiment 2, only reversing the order of presentation so that the probe item in Experiment 2 now formed the target for the search, and the memory display now formed the search display. Thus, perceptual load should be closely matched across experiments, but only Experiment 2 should involve memory for multiple items.

\section{Method}

Participants. Fourteen participants who were naive to the experimental purpose were tested ( 11 females; mean age $=20.8$ years; range 19-24 years). All had normal (self-reported) or corrected-tonormal visual acuity and normal color vision.

Procedure. The method was similar to that used in Experiment 2, except that only one object (i.e., two conjoined colors in the form of either a diamond or a parallelogram) was displayed first for $100 \mathrm{msec}$ at the center of the screen, followed by the 900 -msec blank interval, and, finally, by three objects for $200 \mathrm{msec}$. The shapes of the three objects could be all different (heterogeneously shaped objects) or identical (homogeneously shaped objects). The task was for the participants to decide as quickly as possible whether the first object (i.e., the "target") was present among the three objects. In the vein of Experiment 2, the two colored triangles that formed the object in the memory display were always present on the test display, but they belonged to two different objects in $50 \%$ of the cases. Trials were randomly presented; it was impossible to predict which type of displays (i.e., heterogeneous vs. homogeneous) would occur at test. After completing 12 practice trials, the participants were given 96 experimental trials (i.e., 2 [display types] $\times 2$ [presence vs. absence of the target] $\times 24$ cases). If shape homogeneity creates interference at the encoding stage, then response times (RTs) should be longer when searching in homogeneously shaped rather than in heterogeneously shaped object displays.

\section{Results}

The results are shown in Figure 5. The percentage of correct responses was $95.09 \%$ and $95.24 \%$ for the heterogeneously shaped and homogeneously shaped objects displays, respectively. A paired-samples $t$ test on sensitivity $\left(A^{\prime}\right)$ failed to show a significant effect of display type $[t(13)=0.140, p>.891]$. The paired-samples $t$ test on correct RTs also failed to show a significant difference between the two types of displays $[t(13)=0.679$, $p>.50]$. Performance did not differ as a function of type of object (i.e., diamond, Parallelogram 1, and Parallelogram $2 ; p>.25$ and $p>.50$ for accuracy and correct RTs, respectively). The results suggest that the encoding of two-colored objects is not specifically affected by the presence of several homogeneously shaped objects in the display. The advantage of heterogeneous over homogeneous displays observed in the change detection task of Experiment 2 cannot be the result of a bias in the initial selection and encoding of the objects.

\section{EXPERIMENT 4}

The goal of this experiment was to examine the role of the test display used in Experiment 2 in generating the heterogeneous object advantage that was observed. Specifically, it may be that the colors are associated equally well for both the heterogeneous and homogeneous displays, but that the shape of the test item provides an additional retrieval cue (Tulving, 1974; Tulving \& Thomson, 1973) that is useful only in the heterogeneous condition. In Experiment 4, we eliminated any possibility of using the shape of the test item to improve performance. The experiment was identical to Experiment 2 except that the test object was always a neutral shape in both the heterogeneous and the homogeneous displays (see Figure 6). If the advantage of heterogeneous over homogeneous displays persists when the shape of the test object is totally irrelevant, then we can conclude that the effect is not caused by the presence of differential shape cues at test.

\section{Method}

Participants. Fourteen participants who were naive to the experimental purpose were tested ( 10 females; mean age $=21.1$ years; range 19-30 years). All had normal (self-reported) or corrected-tonormal visual acuity and normal color vision. 


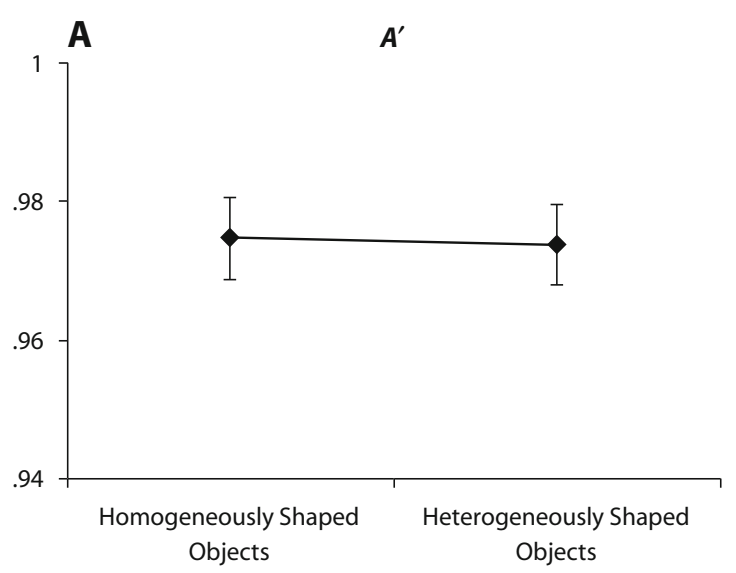

B

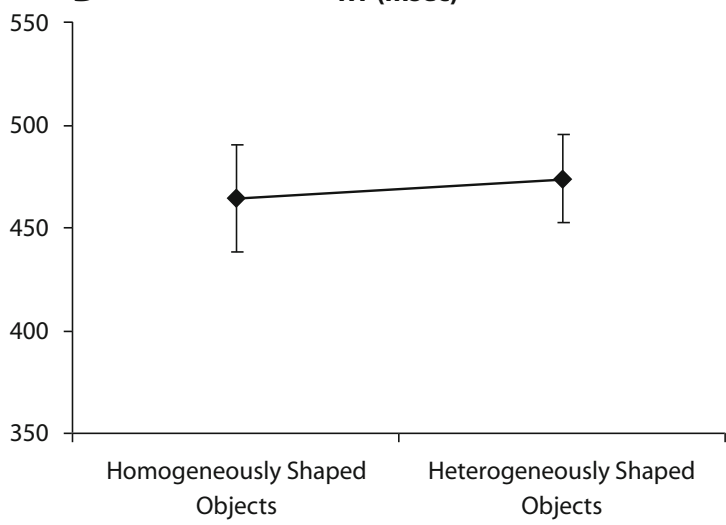

Figure 5. Results of Experiment 3. Error bars represent standard errors of the means.

Procedure. The procedure was similar to that used in Experiment 2, except that in both the homogeneous and heterogeneous displays, two colored disks rather than two colored triangles formed the test object: a small disk subtending $0.95^{\circ}$ surrounded by a larger disk that subtended $1.43^{\circ}$ (see Figure 6). The task was to decide whether the two colors-represented by the disks - were also associated in the previous memory array. The colors in the test display were always present in the memory display, but they came from two different objects in $50 \%$ of the cases. Trials were blocked by display types, for which the order was counterbalanced across participants. After completing 12 practice trials, the participants were given 96 experimental trials (i.e., 2 [display types] $\times 2$ [change vs. no-change trials] $\times 24$ cases $)$.

\section{Results}

The results are shown in Figure 7. A paired-samples $t$ test on sensitivity $\left(A^{\prime}\right)$ revealed a significant effect of display type $[t(13)=2.20, p<.05]$, with better performance in the heterogeneously shaped than in the homogeneously shaped objects displays. A repeated measures ANOVA also tested the effects of object type (i.e., diamond, Parallelogram 1, and Parallelogram 2) in the homogeneously shaped objects condition. The effect was not significant $\left[F(2,26)=0.259, M S_{\mathrm{e}}=0.023, p>.774\right]$. These results

show that the heterogeneous displays advantage still exists, even when the shape of the object at test is totally irrelevant and cannot influence the decision process. We propose that the effect is likely to be due to the way in which the colors and their associations are represented in memory. The quality of the representation of the association between colors is superior when they are conjoined into different shapes. In the General Discussion, we will offer further consideration of the nature of the influence of shape information on color-color associations.

\section{GENERAL DISCUSSION}

The present study addressed a new issue in the VSTM literature regarding whether the similarity between the shapes of objects defined by multiple features from the same dimension affects memory for the features and their combinations. Features (i.e., colors) were paired with homogeneous or heterogeneous shapes. Although the distinctiveness of the shapes does not affect the number of colors that can be maintained simultaneously in VSTM (Experiment 1), it does benefit short-term memory for color associations (Experiment 2). Memory for the pairs of colors that were associated with the same shape was more accurate when the shapes were heterogeneous than when they were homogeneous. This effect does not appear to be caused (1) by greater interference at encoding in the homogeneous displays (Experiment 3 ) or (2) by an additional cue that the shape of the test object may provide at retrieval (Experiment 4).

\section{A Homogeneously Shaped Objects}

Memory Display

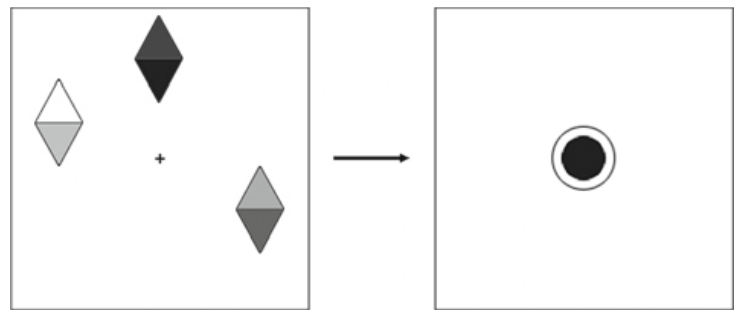

B Heterogeneously Shaped Objects

Memory Display

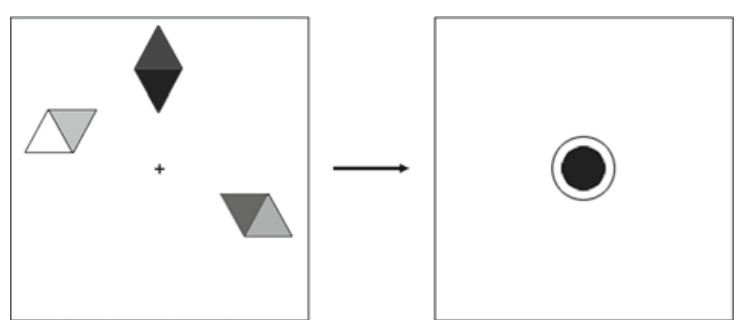

Figure 6. Samples of displays used in Experiment 4. 


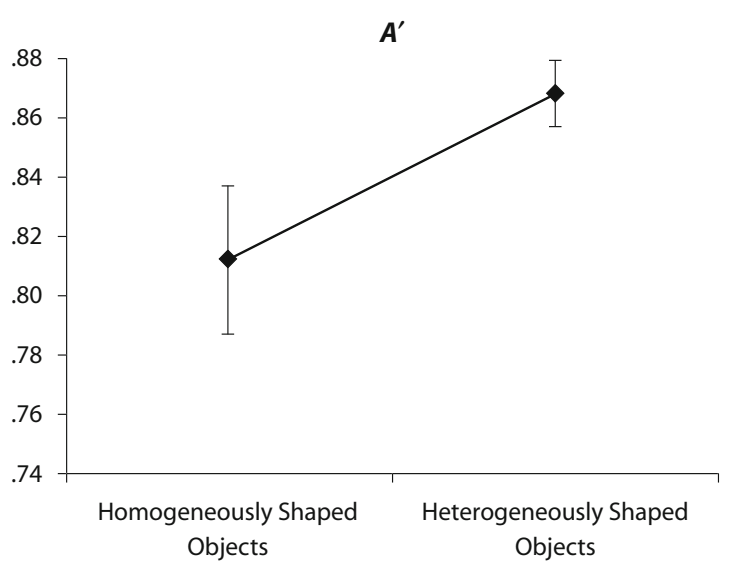

Figure 7. Results of Experiment 4. Error bars represent standard errors of the means.

Before going on to consider the mechanism for the effect of shape distinctiveness on memory for color associations, it is important that we rule out any mundane account. In Experiment 1, in which we assessed memory for color values, we used a whole probe display, whereas in Experiments 2 and 4, in which we assessed memory for color associations, we used a single central probe. Could it be that the presence of multiple items at test interferes with the subtle shape distinctiveness effect? There are two main reasons why we think this is not the case. First, Wheeler and Treisman (2002) showed that the capacity to retain color values in a similar task was not impaired by a whole probe display. Thus, there is little reason to expect that a whole probe display in the present experiment results in interference with memory for the colors. Second, Xu (2002a, 2002b, 2006) showed that various kinds of manipulation of objecthood - including distance and connectedness - can improve memory for the features present in displays, even when tested with whole displays. Indeed, the conditions of Experiment 1 were intended to be close to the conditions employed by Xu (2006), since these are the conditions that are known to produce grouping benefits when features come from different dimensions. An additional difference between Experiment 1 and Experiments 2 and 4 concerns the presence of the separated features condition in Experiment 1. Could it be that recent experience of seeing the triangles apart from one another encouraged participants to treat them separately in the conjoined displays? Again, we think that this is most unlikely. In the experiments of Xu (2002a, 2002b, 2006), separated features conditions are frequently used at no impediment to the object-based grouping benefit.

The present study suggests that the capacity to maintain the associations between colors in memory may be mediated by the association between colors and shapes. When individuals must retain the specific associations between a set of colors grouped into objects, increases in the distinctiveness of shapes aid memory for color associations. To the extent that shapes are distinctive, the association between colors and shapes may become stronger. It ap- pears that direct associations between the colors of objects are rather weak; if the direct associations had been strong, then one would expect little opportunity for modulation by shape differences. Thus, shape memory plays a possible mediating role for color memory.

Previous studies have concentrated on spatial factors as cues to grouping features in memory (e.g., Xu, 2002a, $2002 b)$. The present work demonstrated that other cues to grouping — such as shape dissimilarity—can also affect memory. Future research is needed to investigate whether the effect of shape dissimilarity on memory revealed in the present study is exclusive to features from the same dimension, or whether it can be observed for features from different dimensions. For example, if color and texture or color and motion were examined as features, would the same results obtain? Or, are different dimension features associated more directly?

The results reported in the present article also converge with those of Wheeler and Treisman (2002) in showing that memory for features and memory for feature associations can be dissociated. Wheeler and Treisman found that only memory for feature associations and not memory for features was impaired by the presence of irrelevant nontarget items at test. In the present article, we showed that the two processes are dissociable with respect to the influence of shape similarity. Had we restricted our assessment of the role of shape similarity in memory to features, we would have concluded that shape similarity was unimportant. Indeed, it is not the case that more color information may be stored in memory if colors are paired with heterogeneous shapes. Considering performance in association memory task forced a rather different conclusion, revealing an effect of shape similarity that would otherwise have been hidden. The present results thus underline the importance of distinguishing between memory for features and memory for feature associations, as argued forcefully by Wheeler and Treisman.

An important issue regarding the difference between the feature and association memory tasks remains. That is, to what extent is the representation formed when individuals do the two tasks the same or differently? One plausible possibility is that when individuals try to retain the sets of bicolored objects, the representation formed is the same; the colors are stored, as is the association. The difference between the two tasks then concerns which aspect of this representation is measured - the colors in one case and the association in the other. Since shape distinctiveness only affects the association, effects are found only when the association is measured. An alternative point of view is that different kinds of representation are formed in each task as a result of the unique demands of each task. When the task is to retain colors, the colors are retained independently because there is no incentive to treat them as objects. In contrast, in the association task, different kinds of representation explicitly coding color associations are formed. On this view, shape distinctiveness might affect the quality of object representations - including their constituent features-but can only do so if they have been formed in the first place. Testing between these alternative views will require further careful experimentation. In this 
regard, it will be of interest to determine whether a shape distinctiveness benefit could be found if color memory was tested interleaved with association memory; only on the latter view would a benefit be predicted.

Assuming a selective effect of shape similarity on memory for color associations, there are several possible mechanistic explanations. One possible mechanistic explanation is neural synchronization. It might be that the associations between features are coded by the synchronized firing of neurons in feature-specific areas of visual cortex (Singer \& Gray, 1995). Luck and Vogel (1997, 1998) have further suggested that capacity in VSTM might be understood - at least in part - in terms of the increased probability of accidental synchronization as memory load is increased. By adding global-shape differences to our stimuli, an additional synchronization signal may be provided, helping to keep separate color representations in synchrony. On this account, direct associations between the two colors of an object are strengthened by the addition of a redundant synchronization signal.

Alternatively, a more traditional cognitive explanation might be given along the following lines. Associations between colors are weak, and direct associations may be absent on occasion. Associations between different colors and a common shape provide an additional source of knowledge that participants may consult if necessary. Participants might not know whether the two colors they see were paired together directly, but they might know that the two colors both connect to a common shape representation. Clearly, further research will be needed to determine exactly which scheme provides the best account of the present data.

\section{AUTHOR NOTE}

This research was supported by the Belgian National Fund for Scientific Research (www.fnrs.be). We thank James Enns and one anonymous reviewer for their constructive comments and suggestions. Address correspondence to J.-F. Delvenne, Institute of Psychological Sciences, University of Leeds, Leeds LS2 9JT, England (e-mail: j.f.delvenne@leeds.ac.uk).

\section{REFERENCES}

Aaronson, D., \& WatTs, B. (1987). Extensions of Grier's computational formulas for $A^{\prime}$ and $B^{\prime \prime}$ to below-chance performance. Psychological Bulletin, 102, 439-442.

Alvarez, G. A., \& Cavanagh, P. (2004). The capacity of visual shortterm memory is set both by visual information load and by number of objects. Psychological Science, 15, 106-111.

BADDELEY, A. (1986). Working memory. Oxford: Oxford University Press.

Biederman, I., \& Ju, G. (1988). Surface versus edge-based determinants of visual recognition. Cognitive Psychology, 20, 38-64.

Clifford, C. W. G., Holcombe, A. O., \& Pearson, J. (2004). Rapid global form binding with loss of associated colors. Journal of Vision, 4, 1090-1101.

Delvenne, J.-F., \& BRUYER, R. (2004). Does visual short-term memory store bound features? Visual Cognition, 11, 1-27.

Duncan, J., \& Humphreys, G. W. (1989). Visual search and stimulus similarity. Psychological Review, 96, 433-458.

Grier, J. B. (1971). Nonparametric indexes for sensitivity and bias: Computing formulas. Psychological Bulletin, 75, 424-429.

HumphreYs, G. W. (2001). A multi-stage account of binding in vision: Neuropsychological evidence. Visual Cognition, 8, 381-410.

Humphreys, G. W., Cinel, C., Wolfe, J., Olson, A., \& Klempen, N. (2000). Fractionating the binding process: Neuropsychological evidence distinguishing binding of form from binding of surface features. Vision Research, 40, 1569-1596.
IRWIN, D. E. (1992). Memory for position and identity across eye movements. Journal of Experimental Psychology: Learning, Memory, \& Cognition, 18, 307-317.

IRWIN, D. E., \& ANDREWS, R. V. (1996). Integration and accumulation of information across saccadic eye movements. In T. Inui \& J. L. McClelland (Eds.), Attention and performance XVI: Information integration in perception and communication (pp. 125-155). Cambridge, MA: MIT Press.

Kanwisher, N. G. (1987). Repetition blindness: Type recognition without token individuation. Cognition, 27, 117-143.

LeE, D., \& Chun, M. M. (2001). What are the units of visual short-term memory, objects or spatial locations? Perception \& Psychophysics, 63, 253-257.

LUCK, S. J., \& VoGEL, E. K. (1997). The capacity of visual working memory for features and conjunctions. Nature, 309, 279-281.

LucK, S. J., \& Vogel, E. K. (1998). Response from Luck and Vogel. Trends in Cognitive Sciences, 2, 78-80.

Macmillan, N. A., \& Creelman, C. D. (1991). Detection theory: A user's guide. Cambridge: Cambridge University Press.

Marr, D., \& Nishihara, H. K. (1978). Representation and recognition of the spatial organization of three-dimensional shapes. Proceedings of the Royal Society B, 200, 269-294.

PASHLER, H. (1988). Familiarity and visual change detection. Perception \& Psychophysics, 44, 369-378.

Price, C. J., \& Humphreys, G. W. (1989). The effects of surface detail on object categorization and naming. Quarterly Journal of Experimental Psychology, 41A, 797-827.

Schyns, P. G. (1998). Diagnostic recognition: Task constraints, object information, and their interactions. Cognition, 67, 147-179.

Singer, W., \& GraY, C. M. (1995). Visual feature integration and the temporal correlation hypothesis. Annual Review of Neuroscience, 18, 555-586.

SPERLING, G. (1960). The information available in brief visual presentation. Psychological Monographs, 74(11, Whole No. 498), 1-29.

Treisman, A. [M.], \& Schmidt, H. (1982). Illusory conjunctions in the perception of objects. Cognitive Psychology, 14, 107-141.

Tulving, E. (1974). Cue-dependent forgetting. American Scientist, 62, 74-82.

Tulving, E., \& Thomson, D. M. (1973). Encoding specificity and retrieval processes in episodic memory. Psychological Review, 80, 352-373.

Vogel, E. K., Woodman, G. F., \& Luck, S. J. (2001). Storage of features, conjunctions, and objects in visual working memory. Journal of Experimental Psychology: Human Perception \& Performance, 27, 92-114.

Wheeler, M. E., \& Treisman, A. M. (2002). Binding in short-term visual memory. Journal of Experimental Psychology: General, 131, 48-64.

Wojciulik, E., Husain, M., Clarke, K., \& Driver, J. (2001). Spatial working memory deficit in unilateral neglect. Neuropsychologia, 39, 390-396.

XU, Y. (2002a). Encoding color and shape from different parts of an object in visual short-term memory. Perception \& Psychophysics, 64, $1260-1280$

XU, Y. (2002b). Limitations of object-based feature encoding in visual short-term memory. Journal of Experimental Psychology: Human Perception \& Performance, 28, 458-468.

$\mathrm{XU}, \mathrm{Y}$. (2006). Understanding the object benefit in visual short-term memory: The roles of feature proximity and connectedness. Perception \& Psychophysics, 68, 815-828.

\section{NOTE}

1. $A^{\prime}$ increases from 0.5 for chance performance to 1.0 for perfect performance (see Macmillan \& Creelman, 1991, for more detailed information regarding $\left.A^{\prime}\right)$. $A^{\prime}$ was calculated following the formula developed by Grier (1971): $A^{\prime}=0.5+[(H-g)(1+H-g) / 4 H(1-g)]$, where $H$ is the rate of correct detection of change (hit rate) and $g$ the rate of incorrect detection of change (guessing rate). When $g$ was greater than $H$, the following formula was used (Aaronson \& Watts, 1987): $A^{\prime}=0.5-$ $[(g-H)(1+g-H) / 4 g(1-H)]$.

(Manuscript received August 31, 2006; revision accepted for publication March 4, 2008.) 